\title{
The Three-Nucleon Force Is Not Made by Nature
}

\author{
W. Glöckle, Bochum and P.U. Sauer, Hannover
}

(Institute for Theoretical Physics at respectively Ruhr-Universität and Universität Hannover)

The nucleon is a composite system. It consists of quarks and gluons and is described by quantum chromodynamics. Consequently, the nucleus should be viewed as an assembly of quarks and gluons. However, nature suggests a grossly simplified picture of the nucleus as a satisfactory approximation for nuclear dynamics: All quark and gluon degrees of freedom are to be collected in the motion of nucleonic clusters, which are then considered as elementary. This is the long-standing classic picture of the nucleus with non composite nucleons as basic building blocks. There is overwhelming evidence for its approximate validity: Experimentally, the nuclear mass is given, within $1 \%$, by the nucleons as nuclear constituents and, theoretically, the classic picture has been highly successful in describing a multitude of nuclear structure and scattering phenomena qualitatively. This classic picture freezes all subnucleonic degrees of freedom. It is often amended to revive at least some of them in an average way by allowing nucleons to exchange mesons and undergo transitions to excited nucleonic states, which one calls isobars. The latter description of the nucleus as a system of nucleons, isobars and mesons is sufficiently detailed for most low- and intermediate-energy nuclear phenomena. The real quarkgluon substructure of the nucleon only gets visible in the nucleus, when highenergy projectiles probe the nucleus with a resolution better than $1 \mathrm{fm}$, the nucleonic size. Thus, depending on the phenomena studied different theoretical pictures of the nucleus are appropriate. In which of the alternative pictures do three-nucleon forces arise? How do they arise?

Consider as an illustrative example the process of Fig. 1 for which the description of the nucleus as a system of

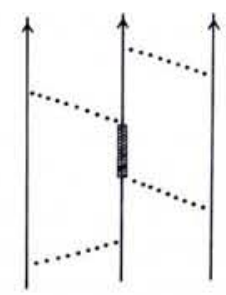

Fig. 1 -Sample process contributing to the interaction in a three-nucleon nucleus. Only its intuitive implication is significant. baryons and mesons is used. The baryons are either nucleons or isobars. Fig. 1 and the diagrams in the following figures, picture the time evolution of the considered system, they are meant to transmit an intuitive understanding of what happens physically. In Fig. 1 the vertical lines stand for the paths of three nucleons in time. Time runs upward and simultaneous events are portayed on the same horizontal level. At some moment nucleon one emits a meson which travels freely to the second nucleon, where it gets absorbed. During this period three nucleons and a meson are present in the system. Later on the exchange of a meson is repeated between nucleons three and two, but the absorption of the meson turns nucleon two into an isobar. The subsequent intermediate state contains a single isobar besides the nucleons. The following interactions now have an obvious meaning. In this description of nuclear dynamics there are no instantaneous two-, three- or manynucleon forces. Instead, there is a mechanism for meson emission and absorption from baryons.

If the meson exchange which lasts a finite time, is assumed to occur instantaneously, i.e., if the meson degrees of freedom are not treated explicitly and only the baryonic ones are kept, one simulates the process of Fig. 1 by the twobaryon interactions of Fig. 2a. They are unretarded and are indicated by horizontal dashed lines. They depend on the coordinates of both baryons and include transition potentials which excite a nucleon to an isobar. If furthermore the isobar is not allowed to propagate a finite time, i.e., if its degree of freedom is also frozen and the physical process of Fig. 1 is considered to occur between nucleons only, an instantaneous effective three-nucleon force arises as shown in the centre of Fig. $2 \mathrm{~b}$. It depends on the coordinates of all three nucleons.

We see, that two-, three or many-nucleon forces are not created by nature as truly fundamental interactions. They arise only when subnucleonic and mesonic degrees of freedom are frozen in a theoretical description of nuclear dynamics. They are an artifact of theoreticians who prefer to work with a restricted number of collective degrees of freedom. But as indicated they do so for good reasons.
Two Different Strategies for Describing the Three-Nucleon Force

By example, Fig. 2 illustrates the strategies for the theoretical description of the physical processes underlying the three-nucleon force. Clearly not all active degrees of freedom can be followed up in a practical calculation. One may either pick out some non-nucleonic degrees of freedom, believed most important, and treat them explicitly. Then the force structure remains comparatively simple as in the example of Fig. 2a, in which two-baryon potentials suffice for the description. Or one decides to work with nucleonic degrees of freedom only, but then one has to face effective threeor, in general, many-nucleon forces explicitly as in the example of Fig. $2 b$. Research is being pursued according to both strategies.

Since there is no full understanding of the two-nucleon force, the understanding of the three-nucleon force must be correspondingly limited. This is why both approaches for the three-nucleon force employ physically incomplete and at present different dynamic input in their practical procedure. They yield only approximative pictures of the threenucleon force, pictures which are even physically unequivalent. They are based on meson exchange. Their small-distance phenomenology will have to be replaced later on by a quark-gluon description as it is developing for the two-nucleon force. Both approaches should and can be enriched finally to contain the same dynamics though in different descriptions.

Both approaches have their advantages and disadvantages. At present, approach one makes the choice of keeping as non-nucleonic degree of freedom

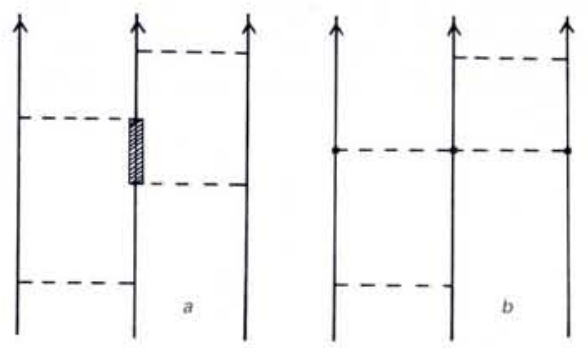

Fig. 2 - Freezing degrees of freedom into instantaneous potentials. In Fig. 2a the meson exchange which lasts a finite time is assumed to occur instantaneously whereas in Fig. 2b, the isobar also is not allowed to propagate over a finite time. 
$\mid$

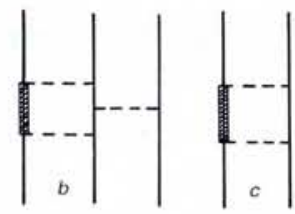

Fig. 3-Sample isobar contributions to effective three-nucleon (Figs. $3 a$ and $3 b$ ) and two-nucleon (Fig. 3c) forces. Consistency requires that all two-and three-nucleon contributions of this type have to be calculated together.

only the $\triangle$-isobar. The $\triangle$-isobar is the lowest-lying excited state of the nucleon, its mass is about $300 \mathrm{MeV}$ heavier than that of the nucleon. It has spin $\frac{3}{2}$ and isospin $\frac{3}{2}$. Allowance for $\triangle$-isobar excitation in nuclear dynamics yields the processes of Figs. $3 a-3 c$ as samples for those considered in approach one. Figs. $3 a$ and $3 b$ represent contributions to the three-nucleon force. They are composed of simpler twobaryon interactions, but cannot be built up as a sequence of two-nucleon potentials. The $\triangle$-isobar is seen predominantly in P-wave pion-nucleon scattering. The central part of Fig. 3a is just such a meson-nucleon scattering process, in which a meson hits a nucleon, excites it into a $\triangle$-isobar, which then propagates and turns itself back into a nucleon by the re-emission of the meson.

Clearly approach one exploits only part of the knowledge on meson-nucleon scattering. In contrast, approach two, which uses nucleonic degrees of freedom only, can absorb much richer physics in its instantaneous threenucleon force. The central shaded area of Fig. 4 is meant to represent all physical process which contribute to meson-nucleon scattering in instantaneous approximation. Among them there is the process with the $\triangle$-isobar as intermediate state on which approach one relies. But other isobars and nucleon-antinucleon pairs can contribute as well as two-meson resonnances which are formed by the in- and outgoing meson and which also couple directly to the nucleon. The latter mechanism is realized by the rho-meson in pion-nucleon scattering. The program

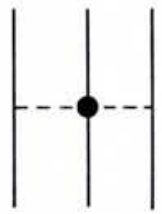

Fig. 4 - Two-meson exchange threenucleon force of approach two. At present only the two-pion exchange is used. It contains the process of Fig. $3 a$ in an instantaneous description besides other two-pion exchange processes. of approach two has been carried out for the two-pion exchange part of the threenucleon force and is being extended to other mesons. Approach one goes easier beyond the level of two-pion exchange by basing the transition potential from nuclear to isobar configurations on any meson exchange or on subnucleonic degrees of freedom.

The effective two- and three-nucleon forces of approach one are energydependent due to isobar propagation as shown in Fig. 3; they feel the presence of other nucleons and can adjust to the nuclear medium. This medium effect turns out to be important for the process of Fig. 3c. Two nucleons interact, and one is thereby excited to a $\triangle$-isobar. After propagation the $\triangle$-isobar is deexcited to a nucleon through a second interaction with the same nucleon. This process also occurs in the isolated twonucleon system; it is a contribution to the two-nucleon force. The presence of the third nucleon, which is of course attached to the other two through interactions - prior and later to the interaction sequence illustrated in Fig. $3 \mathrm{c}$ and therefore not shown there - modifies the propagation between the two transition potentials. The attraction is reduced in bound systems, the medium effect of Fig. $3 c$ on the two-nucleon force is effectively repulsive. In contrast, the twoand three-nucleon forces of approach two are in its present form instantaneous and do not adjust to the surrounding nuclear medium. In approach one there is a worked-out consistency between two-and three-nucleon forces and corrections for electromagnetic and weak currents. The corresponding consistency in approach two has not been studied yet.

\section{What Does the Three-Nucleon Force Look Like?}

One has a good picturesque understanding of the structure of the twonucleon force. Its short range repulsion is followed by the intermediate-range attraction which has the long-range onepion exchange tail. In contrast, the three-nucleon potential is a highly complicated object. It depends on the coordinates, spins and isospins of all three nucleons. It is hard to visualize. We attempt to do so for that part of the twopion exchange three-nucleon force of Fig. 4 in approach two, which is generated by $\mathrm{P}$-wave pion-nucleon scattering and which corresponds to the process of Fig. 3a in approach one mediated by the $\triangle$-isobar. This part of the threenucleon force exhibits a strong and physically important spin-isospin depen- dence. This dependence is - for the purpose of a more transparent graphical presentation - averaged out. The averaging is carried out according to a totally antisymmetric spin-isospin dependence which is in the three-nucleon bound state the wave function part of overwhelming weight.

Important geometrical configurations are indicated in Fig. 5. A sample contour plot of the resulting spatial distribution of the three-nucleon potential energy is shown in Fig. 6. It demonstrates the attraction of the three-nucleon force when the three nucleons are in an equilateral triangle configuration. The force becomes repulsive when the nucleons lie on a straight line. This interesting distribution of attraction and repulsion in the three-nucleon force is distinct from the three-nucleon potential energy of the two-nucleon pair interactions. In the latter case, there remains a strong attraction also in the collinear configuration. It is obvious that more insight into the geometrical properties of the full threenucleon force with the inclusion of spinisospin dependence is badly needed for intuitive estimates of its importance.
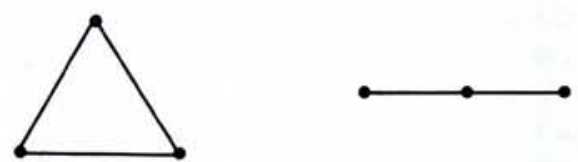

Fig. 5 - Distinct geometrical configurations of three nucleons.

\section{Evidence for the Three-Nucleon Force}

Effective three-body forces are not particular to nuclear physics. Historically, nuclear theory is modelled after the successful quantum-mechanical description of atomic phenomena. There, the photon degrees of freedom are usually frozen into electromagnetic potentials, and two-body interactions seem to suffice for a quantitative account of experimental data. However, three-body forces arise conceptually as shown in Fig. 7. They are tiny indeed due to the small electromagnetic coupling constant and are therefore unimportant in atomic physics. In other systems three-body forces can be sizeable. On the subnucleonic level the confining quark forces resulting from gluon elimination must be very strong and manybody in nature. On a more macroscopic scale, the electron degrees of freedom are often suppressed in chemical. problems. Due to the polarizability of the electron clouds the interaction between two atomic clusters depends on the presence of other atoms, and substantial three-atomic forces emerge. Thus, depending on the system three-body for- 


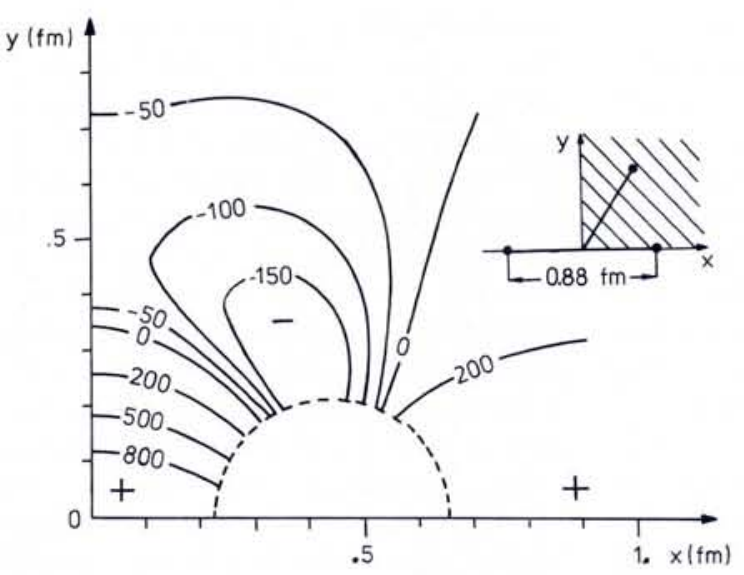

Fig. 6 - Contour plot of the spatial distribution of the three-nucleon potential energy where the two nucleons are kept at a fixed distance of 0.88 $\mathrm{fm}$. The position of the third nucleon is varied according to the insert; contour values are given in MeV. The geometrical configuration of strongest attraction is shielded by the repulsive core of the two-nucleon force.

ces may be small or large. What is the size of the three-nucleon force? What is the evidence for its importance?

The intuitive theoretical estimates on the size of the three-nucleon force are inconclusive and appear even contradictory: The three-nucleon force would derive its possible importance from configurations in which three nucleons are simultaneously close. However, two nucleons strongly repel each other at small relative distances. Consistent with Fig. 6 , the configuration in which the strongest attraction could be gained from the three-nucleon force, is shielded by repulsion due to the two-nucleon potential. This is the reason why effects of the three-nucleon force have often been argued to be small. If, on the other hand, nucleons are spatially rather extended quark-gluon clusters which strongly overlap in the nuclear medium, the three-nucleon force cannot easily be dismissed as unimportant. Obviously our present theoretical understanding of the three-nucleon force is incomplete. One should therefore look for quasi-experimental evidence on the importance of the three-nucleon force. Unfortunately, this evidence is rather indirect.

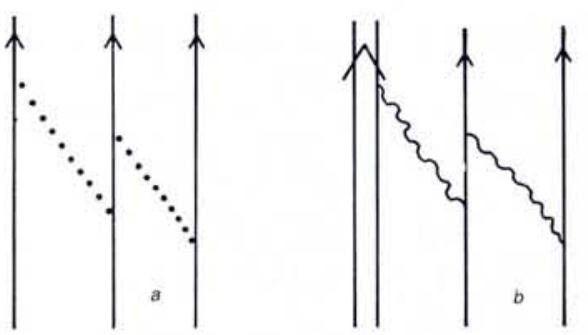

Fig. 7 - Particular two-meson (Fig. 7a) and two-photon (Fig. $7 b$ ) processes contributing to the interaction in the three-nucleon nucleus and in the helium atom where the solid lines indicate electrons, the double line the helium nucleus and the wavy lines the photons. The processes contain intermediate states with two pions and two photons. Freezing the meson and photon degrees of freedom yields intantaneous threebody forces.
Quasi-experimental evidence is obtained from the failure of traditional nuclear theory to explain experimental data. The classic format of nuclear theory views the nucleus as a system of nucleons only, which interact through two-nucleon potentials without the three-nucleon force according to the rules of nonrelativistic quantum mechanics and which couple to the photon through one-nucleon currents. Within this format, the solution of the manynucleon problem is a complex task. It has been accomplished with satisfying numerical accuracy only for some isolated states in a few nuclear systems, i.e., in the three-nucleon system, in the light doubly closed-shell nuclei, in some simple shell-model nuclei and in nuclear matter.

The numerical solution is by far best checked and technically most advanced in the three-nucleon bound state. Irrespective of the particular choice of the two-nucleon potential off-shell, the classic format cannot account for the experimental data quantitatively. In bound systems it is unable to describe simultaneously nuclear binding and nuclear size. The theoretically calculated electromagnetic properties turn out to be in poor agreement with the experimental data. The failure of traditional nuclear theory is documented in the Table. Scattering states can be calculated with confidence only for the threenucleon system, and some encouraging gross agreement with experiment has been obtained. However, two striking problems exist. First, for all realistic twonucleon potentials the resulting zeroenergy neutron-deuteron doublet scattering length turns out to be too large by more than $0.5 \mathrm{fm}$. This discrepancy is correlated to the binding-energy problem. Secondly, the 'So neutron-neutron scattering length, which cannot be measured directly in a scattering experiment, has been extracted from the interaction of two neutrons in the final state through a theoretical model-dependent analysis. The deuteron break-up by a neutron, i.e., $\mathrm{n}+\mathrm{d} \rightarrow(\mathrm{nn})+\mathrm{p}$, and by pion capture, i.e., $\pi^{-}+d \rightarrow(n n)+\gamma$ yield two different values: $-16.3 \pm 0.6 \mathrm{fm}$ and $-18.6 \pm 0.5 \mathrm{fm}$. If the inconsistency in the extracted values would survive additional experimental scrutiny, the inconsistency has to be related to the fact that both reactions differ by the number of hadronic bodies in the final state and may signal the working of the three-nucleon force.

It is tempting to interpret the indicated failures and inconsistencies as due to the missing of non-nucleonic degrees of freedom in the traditional nuclear theory with two-nucleon forces. The added non-nucleonic degrees of freedom will yield many-nucleon forces and manynucleon currents. At present, one can also not exclude the possibility that part of the existing discrepancies is not related to unaccounted dynamical degrees of freedom but rather to the relativistically improper non-covariant treatment of the nuclear kinematics. In any case the existing discrepancies between theory and experiment are often not dramatic, e.g., for the binding energies, the discrepancy is of the order of a few percent in the potential energy. The discrepancies only ask for a modest correction of traditional nuclear theory, e.g., for a small effect due to the three-nucleon force. A complete replacement of the traditional picture of the nucleus is not at all indicated.

\section{Why is Research on the Three-Nucleon Force Exciting?}

Building models is a large part of nuclear theory. There are many extremely useful phenomenological models which describe selected aspects of the nuclear many-body problem qualitatively. The models have a number of parameters, e.g., effective masses, effective charges, effective potentials, which are usually not derived in a microscopic sense from the underlying dynamics of the nuclear constituents. Research on the three-nucleon force is different. It is part of our search for a microscopic theory of nuclear phenomena which is practicable and quantitatively successful.

A microscopic nuclear theory is more ambitious but more limited than phenomenological models and also remains a model. It is more ambitious, since it is more fundamental. It is much more limited in its range and ease of applicability, since it is technically very demanding. The ideas on an improved microscopic theory can at best be tested in simple systems for simple observables, observables, which often appear physically 
${ }^{3} \mathrm{H}$ and ${ }^{4} \mathrm{He}$ binding energies and properties

\begin{tabular}{|l|c|l|}
\hline & \multicolumn{1}{|c|}{ Theory } & \multicolumn{1}{c|}{ Experiment } \\
\hline$E\left({ }^{3} \mathrm{H}\right), \mathrm{MeV}$ & $-7.3 \pm 0.3$ & -8.5 \\
$E\left({ }^{4} \mathrm{He}\right), \mathrm{MeV}$ & $-22 \pm 2$ & -28.3 \\
\hline nuclear & & \\
matter: & & \\
$E / A, \mathrm{MeV}$ & $-17 \pm 2$ & $-15.5 \pm 0.5$ \\
$k_{F}, \mathrm{fm}^{-1}$ & $1.6 \pm 0.2$ & $1.33 \pm 0.04$ \\
\hline
\end{tabular}

The results refer to calculations within the classic format of microscopic nuclear structure employing two-nucleon forces. The quoted values are obtained for the Reid softcore potential. In the theory column the spread of values indicates numerical inaccuracies and variations due to the use of different two-nucleon potentials. The numerical error is smallest for the triton calculation.

unexciting compared to the wealth of nuclear phenomena. Clearly, heavy-ion scattering of ${ }^{16} \mathrm{O}$ from ${ }^{40} \mathrm{Ca}$ or the fission barrier in ${ }^{230} \mathrm{Th}$ are not appropriate testing grounds.

The heavy technique inherent in any microscopic theory blurs handwaving physics. For understanding complicated properties of nuclear systems it may be unnecessary and even undesirable to attempt the connection with the underlying forces. Finally, any microscopic theory of nuclear phenomena is bound to be a simplifying theory, since the full account of all quark-gluon degrees of freedom will not be possible. Even the envisaged microscopic nuclear theory with the three-nucleon force is after all only a model. However, it is a unifying model providing the conceptual basis on which phenomenological descriptions rest, and it is therefore microscopic in the nuclear physics sense.
What has been established in the research on the three-nucleon force? Binding energies and electromagnetic properties of some nuclear bound states and simple scattering states have been calculated microscopically with the inclusion of the three-nucleon force. Approach one, which keeps some subnucleonic degrees of freedom in the description of nuclear phenomena as in Fig. 3 , and approach two, which works with nucleons only and the explicit threenucleon force of Fig. 4, have been used in the three-nucleon bound state. Approach two is being applied to the threenucleon scattering states, the fournucleon problem and nuclear matter. All calculations still have to mature technically.

The resulting effects of the threenucleon force or the effects of the dynamics underlying the three-nucleon force are usually beneficial, although no quantitative success in removing long-standing theoretical problems can be claimed. The three-nucleon force yields additional binding as required. The two-pion three-nucleon force increases the binding in ${ }^{3} \mathrm{H}$ by $-1.1 \mathrm{MeV}$, in ${ }^{4} \mathrm{He}$ by $-7.2 \mathrm{MeV}$. It overbinds nuclear matter at too high a density. This behaviour is expected, since the force lacks any short-range dynamical modification. In contrast, balancing repulsive mechanisms are seen in approach one, which may stabilise the binding-energy results for higher densities. Despite the different physics content, the dominating part of the three-nucleon force, indicated in Fig. 3a, yields additional binding for the threenucleon bound state, comparable in size to the value of approach two. Besides the attraction there are two repulsive mechanisms incorporated in approach one: (i) the rho-exchange screens part of the attraction arising from the pionexchange for single-isobar excitation; (ii) the isobar is allowed to propagate in the nuclear medium thereby decreasing the effect of the three-nucleon force and the attraction of the two-nucleon interaction as pointed out before. Compared to a description with frozen isobar degrees of freedom, repulsion results. Approach one always aims at a consistent description of other nuclear observables besides binding energies and finds there the effect of the mechanisms yielding the three-nucleon force disappointingly small.

Building an effective theory, though microscopic in the nuclear sense, is a slow process. The first results on the three-nucleon force indicate that we are only at the beginning of a long road. The road will lead through the territory of mesonic and subnucleonic degrees of freedom and connects nuclear phenomena with insights of particle physics. The new territory is exciting for nuclear physics. Exploting this territory is an important intellectual challenge. The threenucleon force is an interesting site in this territory.

\section{Suggested Reading}

1. Friar J.L., Three-Body Forces, AIP Conference Proceedings $N^{\circ} 97$ "The Interaction between Medium Energy Nucleons in Nuclei-1982", ed. H.O. Meyer, p. 378.

2. Friar J.L., Gibson B.F. and Payne G.L., ThreeNucleon Forces and Meson-Exchange Currents in the Trinucleon, Comments Nucl. Part. Phys. 11(1983) 51.

3. Sauer P.U., The Three-Nucleon Bound State with Isobaric and Pionic Degrees of Freedom, Nuovo Cim. 76A (1983) 309.

4. McKellar B.H.J. and Glöckle W., ThreeNucleon Forces, Proceedings of the 10th International Conference on Few Body Problems in Physics, ed. B. Zeitnitz.

\section{New Members of the European Physical Society}

\section{Category 4c}

Belgian Physical Society

N. Heymans,

Wezembeek-Oppem

R. Koch, Welkenraedt

J. Van Der Rest, Liège

\section{Eötvös Lorand Physical} Society

L.P. Csernai, Budapest

A. Földes, Budapest

Z. Füzessy, Budapest

French Physical Society

J. Fleury, Talence

J.-B. Theeten, Limeil-Brevannes

\author{
Finnish Physical Society \\ H.K.S. Collan, Espoo \\ K.V. Heikkilä, Helsinki \\ J.P. Hirvonen, Vantaa \\ A.J. Kallio, Oulu \\ T. Makkonen, Helsinki \\ S. Penttilä, Pori \\ E.J. Pinn, Porvoo
}

German Physical Society

K.-P. Doerpelkus, Stolberg

H. Vernickel, Garching

W. Weber, Karlsruhe
Hellenic Physical Society

N. Frangis, Thessaloniki

C. Manolikas, Thessaloniki

S. Sgouridis, Graz, A

D. Zevgolis, Thessaloniki

The Institute of Physics

R.J.A. Bradley, Skerries, IRL

S.M. Juma, Birmingham

Italian Physical Society

R. Daniele, Palermo

G. Vitiello, Salerno

Polish Physical Society

A. Kopystynska, Warsaw

A. Paja, Krakow

S.G. Rohozinski, Warsaw

K. Sienicki, Gdansk
Spanish Royal Society of Physics

J.A. Artiz-Cohen, Madrid

P.L. Garcia-Ybarra, Madrid

J. Jimenez, Madrid

M. Rubio, Madrid

A. Ruiz de Elvira,

Alcalà de Henares

Swiss Physical Society

J.-P. Derendinger, Geneva

\section{Category 4)}

(American Physical Society)

R. Hefferlin, Collegedale, TN

W.D. Knight, Berkeley, CA

K. Kumar, Cookeville, TN

N.D. Lang, Yorktown Heights, NY

K.-F. Liu, Lexington, KY

J. Lyon, Oak Ridge, TN 